\title{
Non-invasive monitoring of blood haemoglobin for analysis of fluid volume kinetics
}

\author{
Robert G. Hahn, Yuhong Li and Joachim Zdolsek
}

\section{Linköping University Post Print}

\section{Tweet}

N.B.: When citing this work, cite the original article.

This is the pre-reviewed version of the following article:

Robert Hahn, Y Li and Johann Zdolsek, Non-invasive monitoring of blood haemoglobin for analysis of fluid volume kinetics, 2010, Acta Anaesthesiologica Scandinavica, (54), 10, 1233 1240 .

which has been published in final form at:

http://dx.doi.org/10.1111/j.1399-6576.2010.02321.x

Copyright: Wiley-Blackwell http://eu.wiley.com/WileyCDA/Brand/id-35.html

Postprint available at: Linköping University Electronic Press http://urn.kb.se/resolve?urn=urn:nbn:se:liu:diva-60717 


\title{
Non-invasive monitoring of blood haemoglobin for analysis of fluid volume kinetics
}

\author{
Robert G. Hahn, Yuhong Li and Joachim Zdolsek \\ Department of Anaesthesia, Faculty of Health Sciences, \\ Linköping University, Linköping, Sweden.
}

Secondary address of Dr. Li: Departments of Anaesthesia, First Affiliated Hospital, College of Medicine, Zhejiang University; Hangzhou, People's Republic of China.

\section{Corresponding author:}

Li Yuhong, MD, PhD

Department of Anaesthesia

Linköping University Hospital

58581 Linköping, Sweden

Phone: +46737272213

Fax: +4613222836

E-mail: yuh_li@hotmail.com

Abbreviated title: Non-invasive hemoglobin 


\begin{abstract}
Background: A commercially available pulse oximeter that reports blood haemoglobin $(\mathrm{Hb})$ concentration is evaluated. This study considers whether this device can deliver serial $\mathrm{Hb}$ data that would be sufficiently reliable for volume kinetic analysis of infusion fluids.

Methods: Forty infusions of $5 \mathrm{ml} / \mathrm{kg}$ or $10 \mathrm{ml} / \mathrm{kg}$ of acetated Ringer's solution were given over $15 \mathrm{~min}$ in 10 healthy volunteers. $\mathrm{Hb}$ was measured on 17 different occasions over 120 min by the Radical 7 pulse oximeter and compared to the result of invasive blood sampling (control). A one-volume kinetic model was applied to each data series. The pulse oximeter also reported the perfusion index.

Results: The median deviation between the 680 invasive and non-invasive $\mathrm{Hb}$ samples (the accuracy) was $1.6 \%$ and the absolute median deviation (precision) was $4.6 \%$. Between-subject factors explained half of the variation in the difference between non-invasive versus invasive sampling.

Ten of the 40 non-invasive series of $\mathrm{Hb}$ values were discarded from kinetic analysis due to poor quality. The remaining 30 series showed a smaller distribution volume for the infused fluid when kinetic analysis was based on the non-invasive method (3.0 versus 5.3 litres; $P<0.001)$. This was due to co-variance with the perfusion index which exaggerated the decrease in $\mathrm{Hb}$ caused by the infusions. The non-invasive method might provide useful kinetic data at the group level, but individual curves deviated too much from the invasive data to be reliable.
\end{abstract}

Conclusions: Non-invasive measurement of the $\mathrm{Hb}$ concentration during volume loading could not provide useful kinetic data for individuals.

Keywords: Equipment, pulse oximeter; Fluids, i.v., Model, pharmacokinetic; 
Pulse oximetry is continuously being developed to provide new parameters of value to the anaesthetist. Besides displaying haemoglobin saturation and heart rate, some modern oximeters now report carbon dioxide pressure, perfusion index and respiratory variations in plethysmographic waveform amplitude. ${ }^{12}$

A pulse oximeter has recently been marketed by Masimo Corporation that has the capacity to measure the blood haemoglobin $(\mathrm{Hb})$ concentration. The device, which is named Radical 7, is easy to use and reasonably priced, although the probes add to the cost by having a short lifespan. In theory, a pulse oximeter with this capability could be used to provide single-point measurements of $\mathrm{Hb}$ that would aid perioperative decisions about, for example, when to transfuse erythrocytes.

Serial analyses of $\mathrm{Hb}$ are also of interest as they can be used to calculate the distribution and elimination kinetics of an infused fluid volume. ${ }^{3-5}$ Volume kinetics has served as a research method for more than a decade ${ }^{6}$ but its clinical use is largely limited by the fact that each analysis requires a long series of sequential $\mathrm{Hb}$ samples. ${ }^{7} \mathrm{~A}$ non-invasive alternative to withdrawal of blood to obtain these types of data could greatly increase the clinical utility of this mathematical approach to fluid therapy.

The present study examines whether serial non-invasive $\mathrm{Hb}$ measurements deliver similar data to those generated by conventional invasive $\mathrm{Hb}$ sampling from venous blood in volunteers undergoing fluid therapy. Our hypothesis was that the new non-invasive monitoring of $\mathrm{Hb}$ would provide data of accuracy and precision high enough to replace invasive blood sampling for volume kinetic analysis. 


\section{Methods}

Between September 2009 and April 2010, 40 experiments were performed in 10 male healthy volunteers aged 19 and 37 years (mean, 22) and with a body weight of between 75 and $100 \mathrm{~kg}$ (mean, 80). The protocol was part of project evaluating the effects of modest dehydration on the kinetics of crystalloid fluid and was approved by the Ethics Committee of Linköping University (Ref. M114-09, ClinicalTrials.gov Indentifier NCT01062776). Each volunteer gave his consent for participation after being informed about the study both orally and in writing.

The experiments started at 9:00 AM in the Department of Intensive Care at Linköping University Hospital. The volunteers had fasted since midnight but had imbibed water at 6:00 AM. They rested on a bed and had an OPN Thermal Ceiling radiant warmer (Aragona Medical, River Vale, NJ, USA) placed about $1 \mathrm{~m}$ above them. The heat was adjusted to achieve maximum comfort for the volunteer.

\section{Experiment groups}

After at least 20 min of rest in the supine position, plasma volume expansion was induced by infusing acetated Ringer's solution intravenously. All 10 volunteers underwent 4 experiments in random order, which were separated by at least two days:

(1) infusion of $5 \mathrm{ml} / \mathrm{kg}$ of acetated Ringer's solution in the euhydrated state,

(2) infusion of $5 \mathrm{ml} / \mathrm{kg}$ of acetated Ringer's solution in the dehydrated state,

(3) infusion of $10 \mathrm{ml} / \mathrm{kg}$ of acetated Ringer's solution in the euhydrated state, and

(4) infusion of $10 \mathrm{ml} / \mathrm{kg}$ of acetated Ringer's solution in the dehydrated state.

All infusions were administered over 15 min via an electronic infusion pump (Volumat MC Agilia, Fresenius Kabi, Bad Homburg, Germany). The infusion time and the volume was chosen to create prompt but modest changes in $\mathrm{Hb}^{7}$

The Ringer's solution had the following ionic content: sodium 130, chloride 110, 
acetate 30 , potassium 4, calcium 2, magnesium $1 \mathrm{mmol}^{-1}$. The fluid bag was kept on the radiant warmer above the bed to maintain a temperature of $27-30^{\circ} \mathrm{C}$ when entering the volunteer.

In experiments 2 and 4, dehydration of 1.5-2 litres was induced by repeated small intravenous doses of $5 \mathrm{mg}$ of furosemide, up to a total of $20 \mathrm{mg}$. The lag time between the last dose of furosemide and the subsequent infusion was $60 \mathrm{~min}$.

\section{Measurements}

A Radical 7 pulse oximeter (Masimo Corp., Irvine, CA, USA) was applied to the index or middle finger of one hand and left in the same place throughout the experiment. A repeated-use probe lasting for 60 hours was used. The apparatus was purchased in May 2009 and used the software delivered by the manufacturer at that time (SET V7.4.0.9, Handheld R.7.7.1.0 and D-station R5.1.2.7).

The Radical 7 displayed the blood haemoglobin $(\mathrm{Hb})$ concentration, perfusion index (PI) and pleth variability index (PVI). The display was set to show $\mathrm{Hb}$ in venous blood, and the data were averaged every 6 seconds.

$\mathrm{PI}$ is the ratio between the pulsatile and non-pulsatile absorption of infrared light at the monitoring site; it represents the pulse strength as the absorption of infrared light is influenced by the amount of blood rather than by the oxygenation. Upon inclusion in the study, the volunteers were tested to have a PI $>2$ because preliminary experiments indicated that peripheral vasoconstriction distorts the non-invasive $\mathrm{Hb}$ analysis.

PVI represents the breath-induced variation in PI; it is calculated as $\left(\mathrm{PI}_{\max }-\mathrm{PI}_{\min }\right) / \mathrm{PI}_{\max }$, over several respiratory cycles. ${ }^{1}$

A cannula was placed in the cubital vein of each arm to infuse fluid and sample blood, respectively. Venous blood $(4 \mathrm{ml})$ was withdrawn with a vacuum tube from the venous cannula every $5 \mathrm{~min}$ during the first $50 \mathrm{~min}$ and then at 60, 70, 90105 and $120 \mathrm{~min}$. The baseline sample was drawn in duplicate, and the mean was used in subsequent kinetic calculations. A total of 17 samples were taken. A small discard volume of blood was drawn before each blood collection to preclude any admixture of rinsing solution, and 2 
$\mathrm{ml}$ of $0.9 \%$ saline was injected after each sample collection to prevent clotting. The venous blood was used to measure the $\mathrm{Hb}$ concentration and the haematocrit on a Cell-Dyn Sapphire (Abbott Diagnostics, Abbott Park, Ill., USA).

The non-invasive $\mathrm{Hb}, \mathrm{PI}$ and PVI, as displayed on the front of the Radical 7, were noted at the same points in time as blood was withdrawn. Haemodynamic monitoring also included non-invasive recording of blood pressure and heart rate.

\section{Kinetic analysis}

Volume kinetic analysis was performed according to a one-volume model. Here, infusion of fluid at the rate $R_{\mathrm{o}}$ expands a functional body fluid volume $V$ to $v$. Elimination occurs by means of baseline fluid losses $\left(\mathrm{Cl}_{\mathrm{o}}\right)$ and a dilution-dependent elimination mechanism (clearance, $\mathrm{Cl} \cdot$ plasma dilution). The differential equation is: ${ }^{3-7}$

$$
\frac{d v}{d t}=R_{o}-C l_{o}-C l \bullet \frac{(v-V)}{V}
$$

Curve-fitting was performed by least-squares regression, programmed into the computer program Matlab 4.2 (Math Works Inc., Natick, MA, USA), and based on the solution to the differential equation shown above. The output was the optimal estimates of $\mathrm{V}$ and $\mathrm{Cl}$, together with their associated standard deviations (SD), which indicated the certainty with which these two parameters could be estimated.

The plasma dilution was used to indicate the volume expansion of $V($ i. e. $(v-V) / V)$ during the infusion experiment. This was calculated as follows: ${ }^{7}$

$$
\frac{v-V}{V}=\frac{\frac{\text { Hbbaseline }}{\text { Hbinfusion }}-1}{1-\text { haematocribaseline }}
$$

When the curve-fitting procedure had been completed, a dilution-time plot for each experiment was drawn by computer, based on the optimal estimates for $\mathrm{V}$ and $\mathrm{Cl}$. Volume-time plots, which indicated how much of the infused volume remained in the volunteer, were created by multiplying the plasma dilution at any time with the calculated 
value of $V$. Hence, $v-V$ was obtained by taking $(v-V) / V) \cdot V$.

\section{Statistical analysis}

The relative percent difference (RPD) was used to describe the difference between the invasive $\left(\mathrm{Hb}_{\text {inv }}\right)$ and non-invasive measurements of $\mathrm{Hb}\left(\mathrm{Hb}_{\text {non }}\right)$ as follows:

$$
\mathrm{RPD}=\frac{\text { Hbinv }- \text { Hbnon }}{(\text { Hbinv }+ \text { Hbnon }) / 2} \bullet 100
$$

The average RPD for many measurements shows the accuracy of the device, i.e. whether a systematic deviation exists between the non-invasive and the invasive $\mathrm{Hb}$ data.

The absolute value of the RPD was used to express the precision, i.e., degree of deviation between the invasive and the non-invasive data points. ${ }^{8}$ This was obtained by giving all RPD values a positive sign.

The degree of correlation of the non-invasive measurements of $\mathrm{Hb}$ with the invasive measurements was also studied by simple and multiple linear regression analysis, where $r=$ correlation coefficient. The invasive samples were regarded as showing the true $\mathrm{Hb}$ level.

Due to skewed distribution, the data for groups were reported as the median and interquartile range limits.

Statistical comparisons of differences between the two modes of measuring $\mathrm{Hb}$ and between sub-groups of data were made by the Wilcoxon's matched-pair test and the Mann-Whitney $U$ test. The various factors contributing to the variability of RPD were studied by analysis of variance (ANOVA) where the partial eta-square showed the proportion of the total variation attributable to each of them. $P<0.05$ was considered statistically significant. 


\section{Results}

The duplicate blood samples obtained at baseline ensured a CV of $0.8 \%$ for the invasive measurement of $\mathrm{Hb}$.

Based on all 680 paired data points, there was a statistically significant difference between the two modes of measuring the Hb concentration $(P<0.0001)$. The overall RPD was $1.6 \%(-2.7,8.4)$ and the absolute RPD was $4.6 \%(2.2,10.0)$. Hence, the non-invasive method showed slightly lower $\mathrm{Hb}$ values than did the invasive method.

The RPD obtained during the infusion experiments tended to be slightly higher than at baseline, but the differences were not statistically significant (Table 1). In both instances, the interquartile range for the absolute RPD showed that the non-invasive $\mathrm{Hb}$ differed more than $7.5 \%$ from the invasive $\mathrm{Hb}$ in half of the paired data points.

There was a statistically significant linear relationship between the invasive and non-invasive $\mathrm{Hb}$ at baseline (Fig. 1A) and during the experiments (Fig. 1B, C).

There was no difference in RPD depending on whether the volunteer received the fluid challenge when being in the euhydrated or dehydrated state. However, the RPD varied greatly between individuals. Four volunteers had an RPD that deviated markedly from the others, their average RPD being 7.8\% which was 10 times higher than for the remaining 6 volunteers. The corresponding absolute RPD for these two groups of 4 and 6 volunteers was $10.1 \%$ and $3.5 \%$, respectively.

Four-way repeated-measures ANOVA was used to quantify how much various factors contributed to the total variability. The volunteer contributed with $63 \%$, the hydration status (euhydrated or dehydrated) with $2 \%$ and the infused fluid volume (being 5 or $10 \mathrm{ml} / \mathrm{kg}$ ) with only $1 \%$. The corresponding fractions for the absolute RPD were $46 \%$, $1 \%$ and $2 \%$, respectively. 


\section{Kinetic analysis}

Ten of the non-invasive curves missed the expected time-course of the plasma dilution (Fig. 2). In these experiments, one or both of the estimates of $V$ and $C l$ were associated with a standard deviation exceeding 50\%. Thus, these curves were discarded because they were considered to provide no real information.

The remaining 30 non-invasive curves, as well as all of the invasive ones, did follow to the expected time-course of the plasma dilution. The estimates of $V$ and $C l$ were consistently associated with standard deviations much smaller than 50\% (Table 2). These curves were considered to provide potentially reliable information.

The size of $V$ was significantly lower in the non-invasive "good" experiments as compared to the corresponding invasive $\mathrm{Hb}$ data (Table 2). On simulation, this difference acted to exaggerate the plasma dilution at the end of the infusion (Fig. 3).

Based on the individual experiments, there was no statistically significant linear relationship between the optimal estimates of either $V$ or $C l$ as obtained by the non-invasive or the invasive method (data not shown). The $25^{\text {th }}$ and $75^{\text {th }}$ percentage limits for the difference between them were 352 and $3147 \mathrm{ml}$ (for $\mathrm{V}$ ) and -41 and 44 (for $\mathrm{Cl}$ ), respectively. Hence, the $25^{\text {th }}-75^{\text {th }}$ percentage ranges, which then include the $50 \%$ of the experiments showing the smallest difference between the two approaches, were as wide as the optimal estimates of $V$ and $C l$ themselves.

Despite this marked variability, Fig. 3 shows that a fairly similar amount of an infusion of $10 \mathrm{ml} / \mathrm{kg}$ of acetated Ringer's solution was estimated to remain in the body at various points in time when simulating a volume-time plot based on the median values of $\mathrm{V}$ and $\mathrm{Cl}$ in Table 2. In contrast, a corresponding simulation of the plasma dilution over time in Fig. 3 showed greater differences.

\section{Perfusion index}

The perfusion index (PI) decreased during the infusion (Fig. 4A). There was an overall inverse linear relationship between PI and the RPD (Fig. 4B). Thus, for decreasing values 
of $\mathrm{PI}$, the non-invasive $\mathrm{Hb}$ showed progressively lower values relative to the invasive $\mathrm{Hb}$ control.

Multiple regression analysis based on all 680 samples showed that the invasive $\mathrm{Hb}$ concentration $(P<0.001)$ and PI $(P<0.001)$ both served as independent significant predictors of the non-invasive $\mathrm{Hb}$. On inclusion of PI, the correlation coefficient for the linear relationship between the two modes of measuring $\mathrm{Hb}$ increased from 0.33 to 0.45 .

\section{Discussion}

Intravenous infusion of fluid induces haemodilution that, through its degree and time course, provides information regarding how the body handles the infused fluid volume. Serial analysis of these types of data is elaborated in the branch of pharmacokinetics referred to as volume kinetics. ${ }^{3-7}$ In this study, invasive measurements of $\mathrm{Hb}$ served as controls and were compared to the readings of the Masimo Radical 7 pulse oximeter, which provides $\mathrm{Hb}$ by a novel non-invasive technology.

At present, based on our findings, the Radical 7 should be used with caution for this purpose. The non-invasive $\mathrm{Hb}$ differed as much as $7.5 \%$ or more from the invasive $\mathrm{Hb}$ in half of the measurements (Table 1). The data obtained by the two methods also showed a poor overall linear relationship (Fig. 1). Despite this disappointment, a systematic difference between the two methods does not preclude successful volume kinetic analysis. The reason is that the input variable, plasma dilution, is calculated based on the change in $\mathrm{Hb}$ from baseline during a single experiment. Moreover, the accuracy and precision of the non-invasive $\mathrm{Hb}$ was much better in $60 \%$ of the volunteers than in the others, suggesting that many high-quality kinetic analyses could still be made. The mechanism for the marked between-subject variability is unclear.

The subsequent kinetic calculations suggested that the non-invasive $\mathrm{Hb}$ data from $25 \%$ of the experiments should be discarded. The robust one-volume kinetic model 
applied here solved all curves, but the model parameters $V$ and $C l$ were estimated with such poor precision that 10 curves provided no real information. Gross inspection of these dilution-time curves also illustrated that the serial $\mathrm{Hb}$ measurements had failed, as they had missed the expected time-course of the haemodilution (Fig. 2 D-F). If such a finding is made we believe that further analysis of the curves should not be carried out.

The remaining 30 "good" non-invasive curves generated acceptable curve-fits, and the model parameters were estimated with a standard deviation much less than $50 \%$ of their optimal values. The parameter estimates for the non-invasive curves had a smaller overall size of $V$ than did the invasive ones, while the difference in $\mathrm{Cl}$ was much smaller and not statistically significant (Table 2). The impact of measuring $\mathrm{Hb}$ non-invasively rather than by blood sampling was further illustrated by computer simulation. The judgement of whether the non-invasive measurements were sufficiently good on a group level is open to debate (Fig. 3). In any event, the curve-fits based on the non-invasive $\mathrm{Hb}$ data are insufficient for the study of volume kinetics in individual subjects. The width of the $25^{\text {th }}-75^{\text {th }}$ percentile ranges for the differences in $\mathrm{V}$ and $\mathrm{Cl}$ obtained with the invasive and non-invasive techniques inspired little confidence.

The interaction between the perfusion index (PI) and the non-invasive $\mathrm{Hb}$ is one reason to why the serial analysis of $\mathrm{Hb}$ by the Radical 7 was, in our opinion, only modestly successful. This has two aspects. On one hand, a low PI in a volunteer lowers the entire series of non-invasive $\mathrm{Hb}$ and increases the RPD for that experiment. However, Table 1 shows that good parameter estimates can be obtained in a volume kinetic analysis despite such a shift of baseline. On the other hand, PI decreased gradually during the infusion, which exaggerated the infusion-induced haemodilution when measured non-invasively (Fig. 3A). The increased haemodilution explains why non-invasive $\mathrm{Hb}$ yielded lower estimates of $V$. The lowering of the PI could be due to mild vasoconstriction induced by the temperature of the infusion fluid, despite the fact that it was warmed (although not body-warm). The decrease in PI might also be an artefact created by the acute but relatively mild haemodilution, but this is less likely since PI changed by the same magnitude for both the infused volumes. 
The Radical 7 can be set to report either the arterial or the venous Hb. The data are obtained by 13 wavelengths directed to the microcirculation in the finger, which is known to have a much lower $\mathrm{Hb}$ than both arterial and venous blood. Exactly how the calculations are being made remains a company secret. From the present study, it is apparent that the Radical 7 might report $\mathrm{Hb}$ values that differ markedly from those obtained by an invasive venous sample. As discussed above, the difference is dependent on PI but mostly on the individual. It is also of note that experiments in which the non-invasive $\mathrm{Hb}$ changes as expected to a fluid challenge report data that are systematically 2.6-2.8 percent higher than the invasive $\mathrm{Hb}$. In contrast, experiments that poorly capture the expected $\mathrm{Hb}$ changes report non-invasive data that are lower than the venous $\mathrm{Hb}$. Hence, a systematic drift to low $\mathrm{Hb}$ values is coupled to the poor responsiveness to induced $\mathrm{Hb}$ changes seen in the "bad" curves. This might suggest that poor signal detection, possibly due to contact problems between apparatus and skin, played a role in these failures.

A limitation of our study is that a complete evaluation of the accuracy and precision of the non-invasive measurement of $\mathrm{Hb}$ should cover a wider range of $\mathrm{Hb}$ values. Here, an acute reduction of the $\mathrm{Hb}$ concentration of up to $15 \%$ was induced by fluid, but it always remained above $115 \mathrm{~g} / 1$. Moreover, our study captures the quality of the delivery of non-invasive $\mathrm{Hb}$ data by the new Radical 7 only during a specific period of the marketing process. Both the hardware and the software of the apparatus are continuously being improved. For example, the manufacturer has recently recommended the use of single-use plastic probes to optimize the probe-skin contact. The apparatus should also be tested in a larger group of subjects during surgery and in those subjected to severe disease, as these scenarios are of interest to our specialty.

In conclusion, non-invasive measurement of $\mathrm{Hb}$ by the Radical 7 pulse oximeter cannot replace invasive blood sampling for volume kinetic analysis. Besides a prominent between-subject variation, the haemodilution is exaggerated during an infusion of fluid because of co-variance with the perfusion index. 
Acknowledgement: Research nurse Susanne Öster and Helén Didriksson assisted during the experiments. The project was supported by a grant from the European Society of Anaesthesiology.

\section{References}

1. Cannesson M, Desebbe O, Rosamel P, Delannoy B, Robin J, Bastien O, Lerhot JJ. Pleth variability index to monitor the respiratory variations in the pulse oximeter plethysmographic waveform amplitude and predict the fluid responsiveness in the operating theatre. $\mathrm{BrJ}$ Anaesth 2008; 101: 200-6

2. Keller G, Cassar E, Desebbe O, Lehot JJ, Cannesson M. Ability of pleth variability index to detect hemodynamic changes induced by passive leg raising in spontaneously breathing volunteers. Critical Care 2008: 12: R37.

3. Norberg Å, Hahn RG, Husong Li, Olsson J, Prough DS, Börsheim E, Wolf S, Minton R, Svensén $\mathrm{CH}$. Population volume kinetics predicts retention of $0.9 \%$ saline infused in awake and isoflurane-anesthetized volunteers. Anesthesiology 2007; 107: 24-32

4. Borup T, Hahn RG, Holte K, Ravn L, Kehlet H. Intraoperative colloid administration increases the clearance of a postoperative fluid load. Acta Anaesthesiol Scand 2009: 53: 311-7

5. Li Y, Hahn RG, Hu Y, Xiang Y, Zhu S. Plasma and renal clearances of lactated Ringer's solution in pediatric and adult patients just before anesthesia is induced. Pediatric Anesthesia 2009: 19: $682-7$

6. Drobin D, Hahn RG. Volume kinetics of Ringer's solution in hypovolemic volunteers. Anesthesiology 1999; 90: 81-91

7. Hahn RG. Volume kinetics for infusion fluids (review). Anesthesiology 2010; 113: 470-81

8. Varvel JR, Donoho DL, Shafer SL. Measuring the predictive performance of computer-controlled infusion pumps. J Biopharm Biopharmaceut 1992; 20: 63-94 


\section{TABLE 1.}

The Relative Percent Difference (RPD) between the non-invasive and invasive measurements of blood $\mathrm{Hb}$.

\begin{tabular}{lcccc}
\hline & All curves & $\begin{array}{c}\text { "Good" } \\
\text { curves }\end{array}$ & $\begin{array}{c}\text { "Bad" } \\
\text { curves }\end{array}$ & $\begin{array}{c}\text { "Good" versus } \\
\text { "bad"* }\end{array}$ \\
& & & & \\
\hline Baseline, N & 80 & 60 & 20 & \\
RPD (\%) & $1.3(-2.6,7.6)$ & $2.6(2.2,10.2)$ & $-1.4(-3.0,1.6)$ & $P<0.02$ \\
Absolute RPD (\%) & $4.2(2.1,9.6)$ & $4.4(2.3,10.2)$ & $3.0(1.5,5.8)$ & NS \\
During the experiments, N & 600 & 450 & 150 & \\
RPD (\%) & $1.6(-2.7,8.4)$ & $2.8(-1.5,10.0)$ & $-1.3(-7.1,3.8)$ & $P<0.0001$ \\
Absolute RPD (\%) & $4.7(2.2,9.9)$ & $4.6(2.1,10.4)$ & $5.0(2.3,9.3)$ & NS \\
& & & & \\
\hline
\end{tabular}

*Statistical analysis made by using the Mann-Whitney U test.

Data are the median and interquartile limits. 


\section{TABLE 2.}

The optimal estimate and the associated standard deviation (SD) for the volume of distribution at baseline $(V)$ and the clearance $(C l)$ in the 30 experiments in which both non-invasive and the invasive $\mathrm{Hb}$ monitoring could be satisfactorily applied.

\begin{tabular}{cccc}
\hline Kinetic parameter & $\begin{array}{c}\text { "Good” non-invasive } \\
\text { curves }\end{array}$ & $\begin{array}{c}\text { Invasive } \\
\text { curves }\end{array}$ & Wilcoxon's test \\
& & & \\
$\mathrm{N}$ & 30 & 30 & \\
$V($ litres $)$ & $3.0(2.5,4.7)$ & $5.3(4.2,5.9)$ & $P<0.001$ \\
$\mathrm{SD}$ & $0.5(0.3,1.0)$ & $0.5(0.4,0.7)$ & \\
$\mathrm{Cl}(\mathrm{ml} / \mathrm{min})$ & $75(44,121)$ & $66(43,145)$ & $\mathrm{NS}$ \\
$\mathrm{SD}$ & $15(10,31)$ & $12(8,17)$ & \\
\hline
\end{tabular}

Data for groups are given as the median and interquartile limits. 

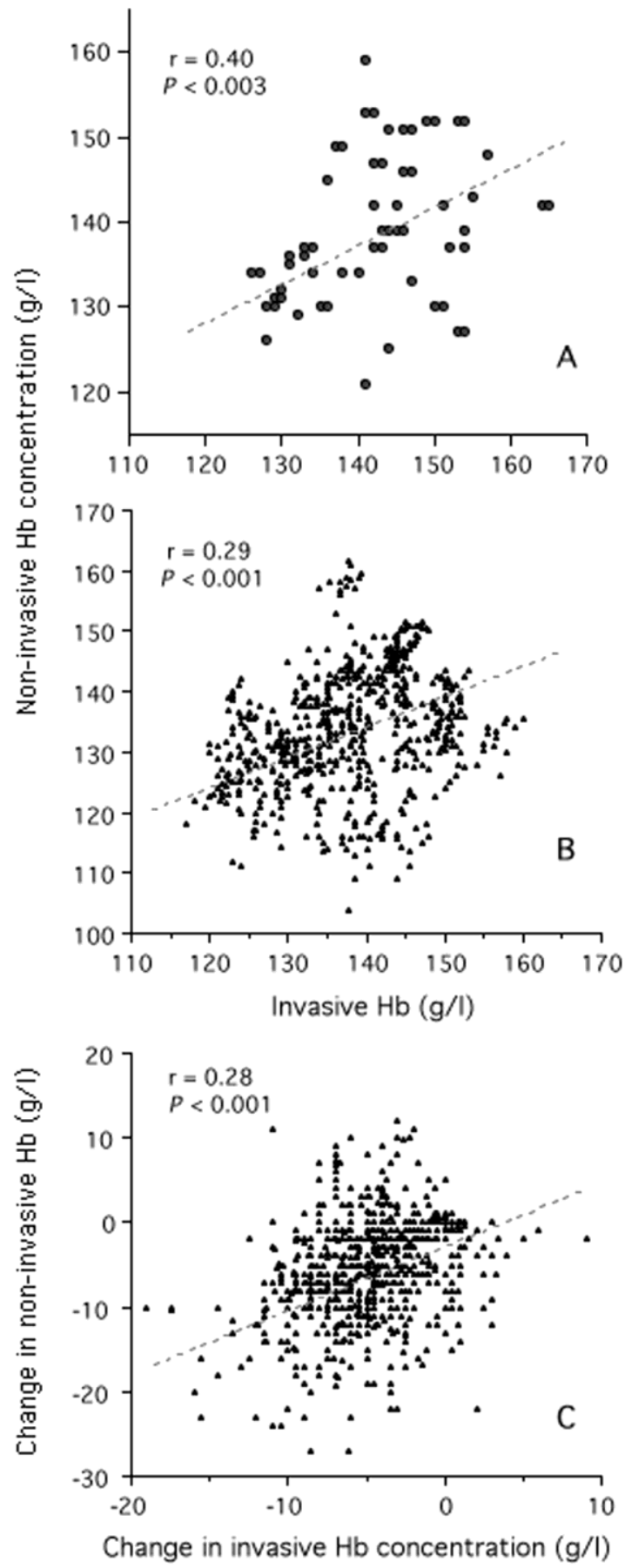

Fig. 1

Relationship between invasive and non-invasive measurements of $\mathrm{Hb}$

$\underline{\text { A: }}$ at baseline, B: during the infusion experiments and C: when expressed as the change from baseline.

where $\mathrm{r}=$ correlation coefficient. 

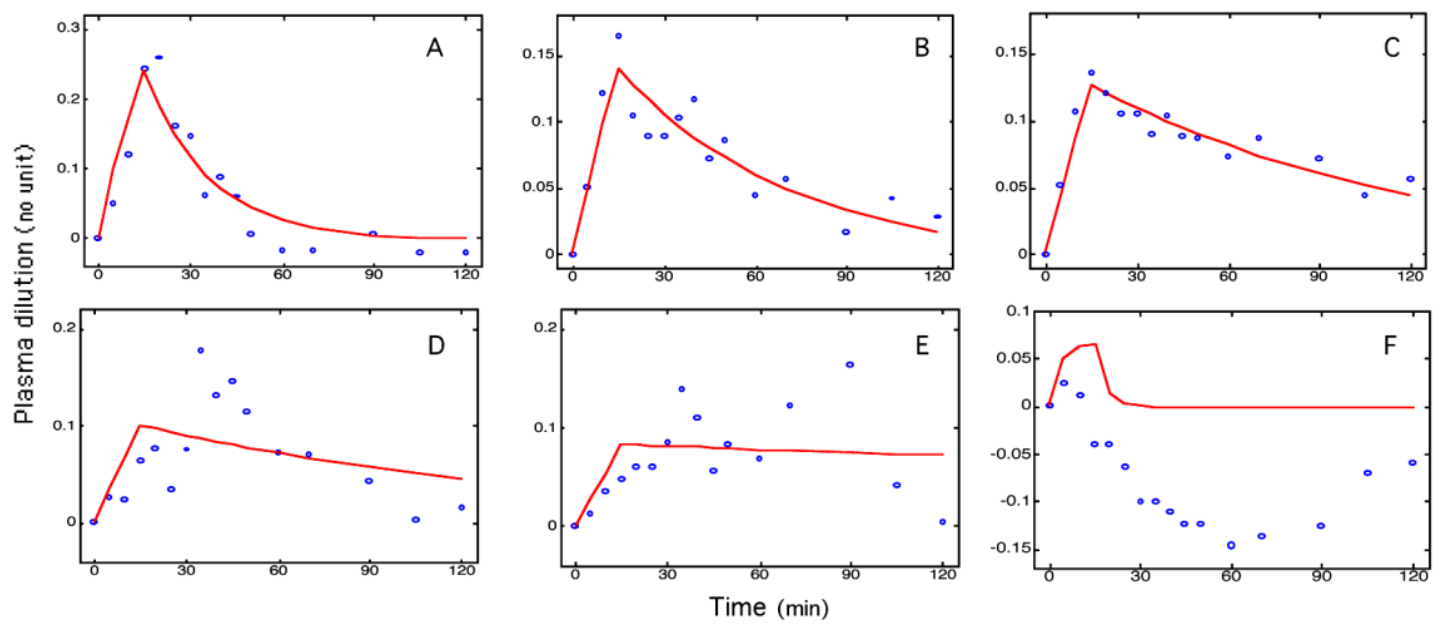

Fig. 2

Non-invasive measurements of the $\mathrm{Hb}$ concentration expressed as the plasma dilution during and after i.v. infusion of acetated Ringer's solution over 15 min in 6 volunteers.

$\underline{\mathbf{A}, \mathbf{B}, \mathbf{C}}$ Curves of good quality.

$\underline{\mathbf{D}, \mathbf{E}, \mathbf{F}}$ Curves of poor quality. 

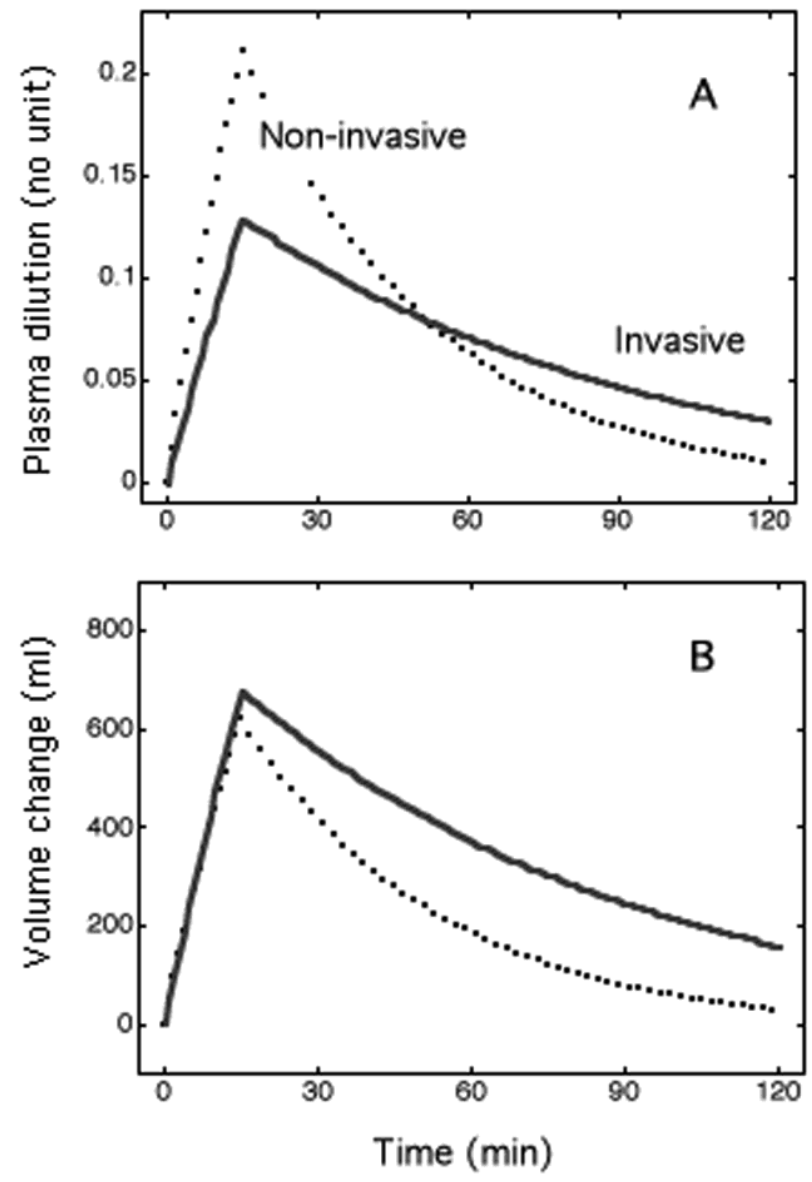

Fig. 3

Computer simulation of $\underline{\mathbf{A}}$ : the plasma dilution and $\underline{\mathbf{B}}$ : the amount of infused fluid remaining in the body. Hypothetical experiments in which $10 \mathrm{ml} / \mathrm{kg}$ of acetated Ringer's solution was infused and where the dilution-time and volume-time curves are based on invasive and non-invasive $\mathrm{Hb}$ measurements. Parameters were derived from Table 2. 

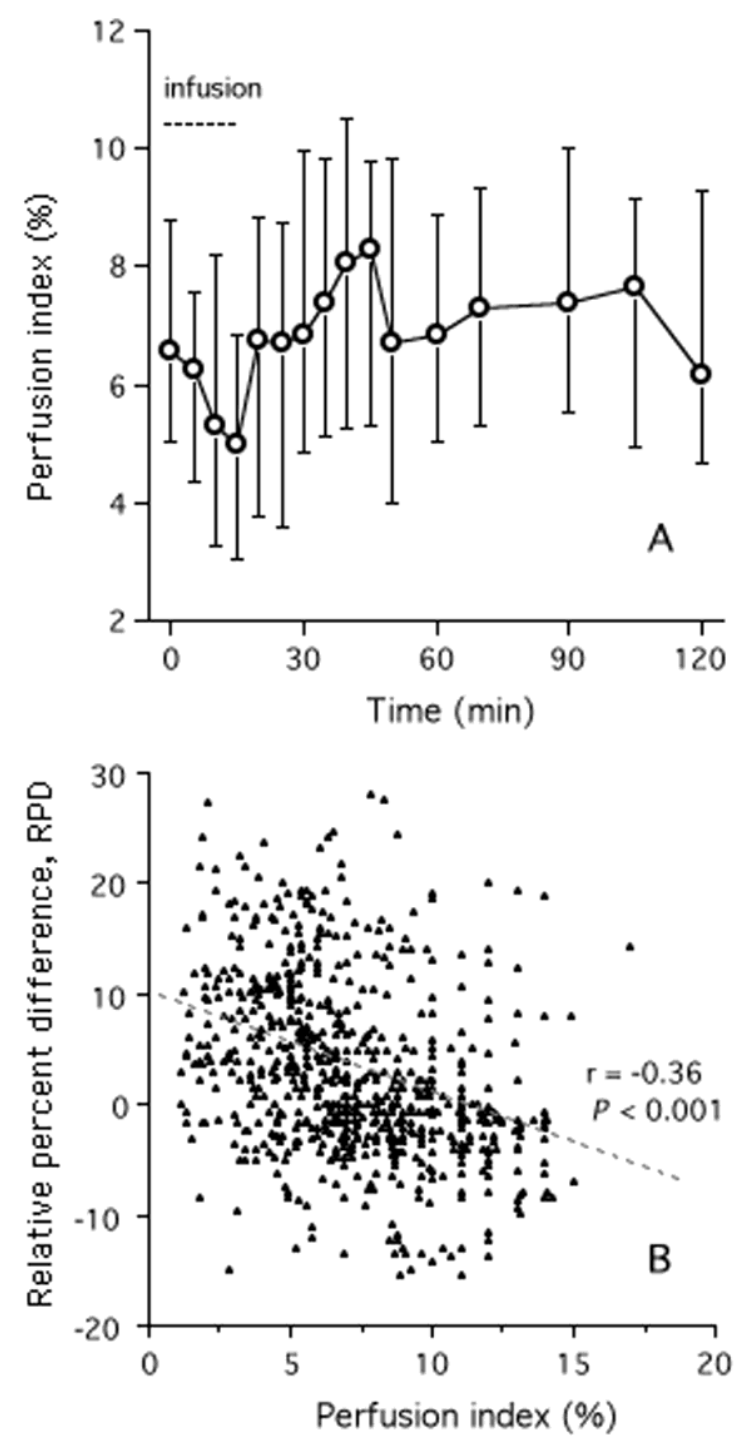

Fig. 4

A: Perfusion index measured by pulse oximeter during and after 40 infusions of acetated Ringer's solution. Data are the median and $25^{\text {th }}-75^{\text {th }}$ percentile range.

B: The relationship between the perfusion index and the relative percent difference (RPD) in $\mathrm{Hb}$ between the invasive and non-invasive technology. Each circle represents one point in time during one of 40 experiments. 